\title{
DOPPLER TRANSCRANEAL EN PRE-ECLAMPSIA
}

Francisco Javier Vargas Malaver MD*

\section{Resumen}

La preeclampsia es una afección multisistémica durante el embarazo, con alta tasa de morbilidad y mortalidad en la madre y el hijo. Dentro del espectro de efectos está la alteración del flujo sanguíneo cerebral que en ocasiones se asocia con convulsiones (eclampsia). Se ha intentado encontrar un método diagnóstico para su detección temprana, iniciar la terapia oportuna y reducir los efectos negativos. El doppler transcraneal es un examen no invasivo, de bajo costo y seguro, que puede tener un valor diagnóstico. El objetivo del artículo es una revisión sobre la utilidad del doppler transcraneal y la preeclampsia. Se buscó en la base de datos PubMed con términos MeSh. Diferentes estudios revelan índices de resistencia y pulsatilidad disminuidos en el segundo trimestre del embarazo, los cuales pueden predecir la aparición de preeclampsia.

Palabras clave: preeclampsia, eclampsia, síndrome HELLP, ultrasonografía doppler transcraneal, vasoespasmo.

Abreviaturas: PRE-E, preeclampsia.

\section{DOPPLER TRANSCRANEAL EN PRE-ECLAMPSIA}

\section{Abstract}

Pre-eclampsia is a multisystem disorder of pregnancy associated with significant maternal and perinatal morbidity and mortality. Among the spectrum of effects are alterations in cerebral blood flow sometimes associated with convulsions (eclampsia). There is a need for a method for diagnosis and early detection of pre-eclampsia to allow timely intervention and reduce negative effects. Transcranial Doppler is a safe, non- invasive, low-cost method which may have a diagnostic value. The objective of this article is to conduct a review on the utility of transcranial Doppler and pre-eclampsia. The PubMed data base was reviewed using MeSh terms. Different studies reveal reduced resistance and pulsatility indices in the second trimester of pregnancy, which may predict the development of pre-eclampsia.

Key words: pre-eclampsia, eclampsia, HELLP syndrome, transcranial Doppler ultrasonography, vasospasm

Fecha recibido: enero 27 de 2014 - Fecha aceptado: junio 20 de 2014

* Residente IV de Radiología e Imágenes Diagnósticas. Fundación Universitaria de Ciencias de la Salud. Bogotá DC, Colombia.
** Asesor académico:Alexandra Bernal MD, Instructor Asociado, Fundación Universitaria de Ciencias de la Salud, Bogotá DC, Colombia. 


\section{Introducción}

La PRE-E se define como el aumento de la presión arterial $(\geq 140 / 90 \mathrm{~mm} / \mathrm{Hg}$ ) después de la semana veinte de gestación, sin historia de elevación previa de la presión arterial, acompañada con frecuencia de proteinuria. ${ }^{1,2} \mathrm{La}$ incidencia es de 2 a $7 \%$ en Norteamerica. ${ }^{3}$ El 70\% de los casos de hipertensión en el embarazo están relacionados con PRE-E. ${ }^{4}$ Es una enfermedad que produce vasoespasmo generalizado con alteración principal en órganos como el cerebro, riñón, hígado y medula ósea; en donde la alteración del endotelio juega un papel principal. ${ }^{5-7}$

Algunos investigadores han sugerido que la disfunción endotelial en el síndrome PRE-E/eclampsia va a producir alteración de la cascada de la coagulación, vasoconstricción y coagulación intravascular que afecta todos los órganos del cuerpo, al igual que a los riñones, y es lo que va a producir la alteración de la hemodinámica cerebral con hipo e hiperperfusión cerebral, siendo esta última frecuente en la PRE-E severa. ${ }^{8-11} \mathrm{Se}$ han propuesto muchos exámenes paraclínicos que puedan predecir la aparición de PRE-E, pero como no se ha logrado encontrar uno de tamizaje que tenga utilidad para lograr este objetivo, es donde el doppler transcraneal puede ser una buena alternativa. ${ }^{12}$ El objetivo principal de este articulo es realizar una revisión narrativa de la literatura para identificar el papel del doppler transcraneal en la detección temprana de la PRE-E, en especial las alteraciones en la autorregulación cerebral que puedan medirse mediante este examen. Se realizó la búsqueda en la base de datos de PubMed utilizando los términos MeSh: ultrasonography, doppler, transcranial pre-eclampsia, con límites de tiempo (entre 1980 y 2013) en artículos en inglés y español. Se encontraron 43 artículos en total, de los cuales solo trece estaban relacionados con el tema.

\section{Alteraciones del parénquima cerebral secundarias a la preeclampsia}

El tejido cerebral puede presentar edema, isquemia y hemorragia como complicaciones secundarias a la PRE-E, al igual que las convulsiones (eclampsia). Los lóbulos parietal y occipital son muy susceptibles a la lesión isquémica o encefalopática. ${ }^{13,14}$ Estos cambios parenquimatosos se conocen como síndrome de encefalopatía reversible posterior (SERP), que tiene incidencia en la circulación posterior por lo cual se ven más afectados los lóbulos occipital y parietal en forma bilateral. Como su nombre lo indica, son cambios reversibles si se realiza un tratamiento temprano..$^{15}$ Los hallazgos en la tomografía computarizada cerebral se presentan en un 50\% de las pacientes, por lo regular hipodensidades transitorias de la sustancia blanca y de la corteza cerebral (Figura 1A); son menos frecuentes los infartos y la hemorragia. ${ }^{16}$ En la resonancia magnética cerebral en secuencias ponderadas en $\mathrm{T} 2$ se identifican hiperintensidades corticales y de la sustancia blanca subcortical relacionadas con edema (Figura 1B). En la angiografía se ha demostrado edema cerebral y vasoespasmo. ${ }^{17}$

\section{Doppler transcraneal}

El doppler transcraneal es un estudio que se realiza con un transductor de $2 \mathrm{MHz}$ a través del cráneo con la finalidad de valorar los vasos intracraneanos (arterias carótida interna, cerebral anterior, cerebral media, vertebral y basilar, entre otras). Para visualizarlos se utilizan diferentes ventanas como son la transtemporal, transorbitaria, suboccipital y submandibular; se realiza una exploración ecográfica en modo B y con doppler color, midiendo las velocidades sistólica máxima y telediastólica, así como los índices de resistencia y de pulsatilidad. ${ }^{18,19}$ Esta última es una propiedad muy importante del flujo, existiendo ondas del doppler de pulsatilidad baja, media y alta. Las arterias carótida interna, vertebrales, renales y celíacas tienen ondas de pulsatilidad baja, debido a que alimentan sistemas circulatorios de baja resistencia al flujo. ${ }^{20,21}$ Cuando se necesita realizar una medición cuantitativa se utilizan las fórmulas de Gosling (Figura 2) para el índice de pulsatilidad y de Purcelot (Figura 3) para el índice de resistencia. ${ }^{22,23}$

Se han descrito múltiples usos del doppler transcraneal dentro de los cuales se incluyen: valoración del vasoespasmo en la hemorragia subaracnoidea, anemia de células falciformes, ateroesclerosis de vasos 

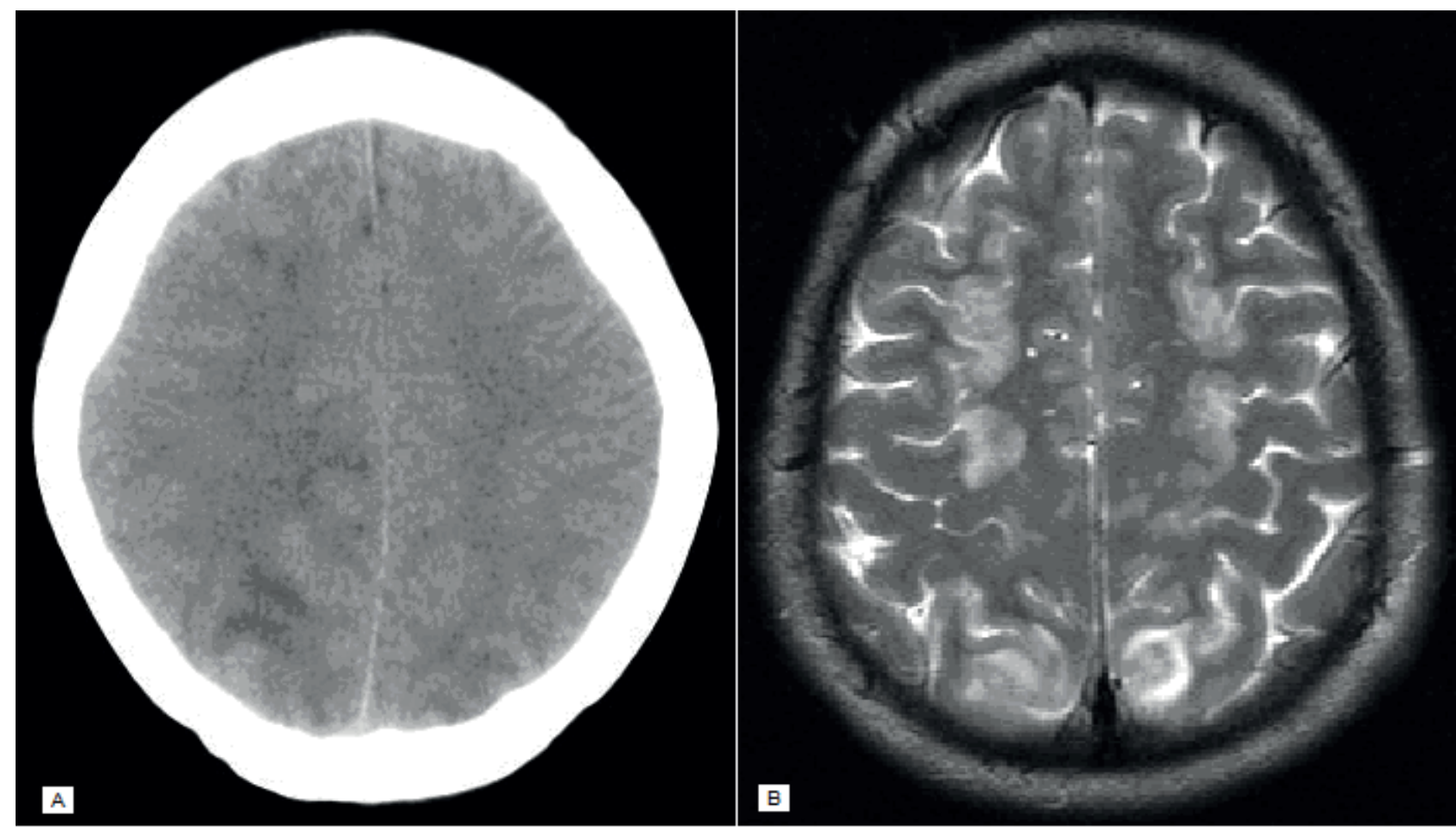

Figuras IA y B. Imágenes de una paciente con diagnóstico de eclampsia. La Figura IA es una tomografía computarizada de cráneo simple que muestra hipodensidades corticales y subcorticales en la región parietal en forma bilateral y simétrica. La Figura IB muestra una resonancia magnética cerebral potenciada en T2 donde se identifican hiperintensidades corticales y de la sustancia blanca subcortical. Cortesía del Dr. German Arango. Neurorradiólogo.

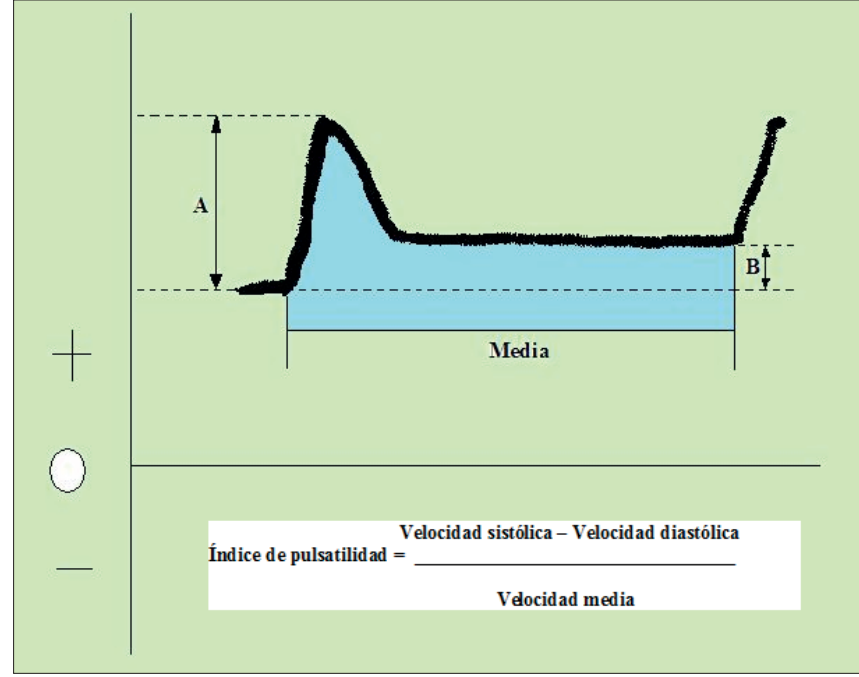

Figura 2. Formula de Gosling. La A representa a la velocidad sistólica y la B la telediástolica. Figura modificada del texto de Zwuebel's Doppler General, Introducción a la ultrasonografia vascular, William J. Zwiebel, John S. Pellerito, Marbán, Elsevier 2008 paginas 59-85 y paginas 20I-222.

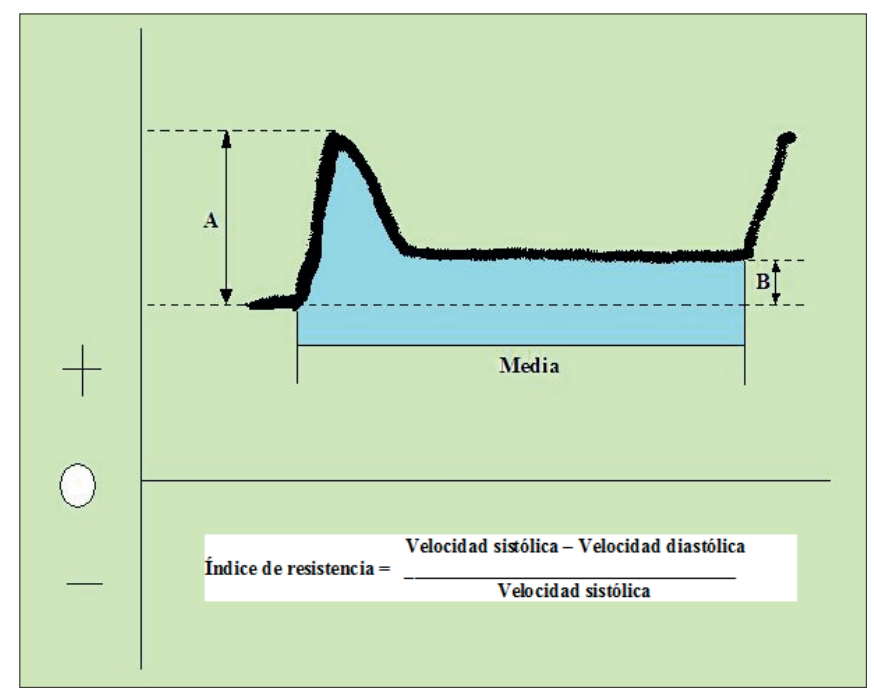

Figura 3. Formula de Purcelot. La A representa la velocidad sistólica y la B la telediástolica. Figura modificada del texto de Zwuebel's Doppler General, Introducción a la ultrasonografia vascular, William J. Zwiebel, John S. Pellerito, Marbán, Elsevier 2008 paginas 59-85 y paginas 20I-222. 
intracraneales, enfermedad cerebro vascular, trauma cráneoencefálico, muerte cerebral, enfermedad arterial carotídea, monitorización transcraneal intraoperatoria, malformaciones arteriovenosas y por supuesto preeclampsia. ${ }^{18}$

\section{Estado hemodinámico y flujo sanguíneo cerebral en el embarazo normal}

Durante el embarazo hay aumento de la poscarga y del volumen plasmático hasta en $40 \%$, alcanzando la semana veinte de gestación con una disminución de la resistencia vascular periférica. ${ }^{24}$ Estos hallazgos están en relación con otros en los cuales hay un estado hiperdinámico materno, con aumento de la poscarga cardíaca y resistencia periférica reducida en etapas tempranas del embarazo. ${ }^{25-27}$ Se ha demostrado que hay un aumento fisiológico del flujo sanguíneo cerebral en el primer trimestre del embarazo por encima del $25 \%$ y que este se reduce poco a poco según va avanzando el embarazo, pero en el tercer trimestre sigue siendo $15 \%$ mayor que el de mujeres normales no embarazadas. ${ }^{28-30}$ Inclusive en un estudio se ha determinado que el flujo sanguíneo cerebral puede elevarse hasta el $52 \%$ del nivel basal durante el embarazo normal. ${ }^{31}$ Bajo condiciones de normalidad hay un sistema de autorregulación que permite que el flujo sanguíneo cerebral no se altere a pesar que ocurra variación de la presión sanguínea sistémica. ${ }^{32}$

Belford y colaboradores identificaron disminución fisiológica de la velocidad sistólica y media de la arteria cerebral en forma lineal durante el embarazo normal, desde la semana doce hasta la cuarenta, al igual que los índices de pulsatilidad y resistencia, mientras la velocidad diastólica no se modificaba en forma importante. Los intervalos de la velocidad sistólica se encontraban entre 80 y $112 \mathrm{~cm} / \mathrm{seg}$, los de la velocidad media de 57 a $81 \mathrm{~cm} / \mathrm{seg}$ y los de la diastólica 39 a 47 $\mathrm{cm} / \mathrm{seg}$. Williams y colaboradores encontraron que la velocidad sistólica de la arteria cerebral media permanecía constante durante los primeros dos trimestres ( 71 $\pm 22 \mathrm{~cm} / \mathrm{seg}$ ), con disminución entre las semanas 29 y 36 de gestación $(62+17 \mathrm{~cm} / \mathrm{seg})$, mientras la velocidad diastólica no tuvo una variación importante. ${ }^{29}$
Pelle y colaboradores encontraron un pico del índice de pulsatilidad en la mitad del embarazo con incremento constante entre las 8 y las 29 semanas de gestación, con velocidad diastólica sin cambios importantes y con un pico de velocidad sistólica a las 15 semanas de embarazo en la arteria cerebral media. ${ }^{24}$

\section{Flujo sanguíneo cerebral en la preeclampsia}

En la PRE-E hay aumento del flujo sanguíneo cerebral posiblemente secundario a vasoconstricción de las arterias basales con vasodilatación de las arteriolas dístales e incremento de la velocidad del flujo sanguíneo de la arteria cerebral media, por lo cual se ha propuesto la hipótesis que hay una alteración en la autorregulación cerebrovascular en mujeres con PREE. ${ }^{32-38}$ Sherman y colaboradores encontraron aumento de la velocidad de flujo de la arteria cerebral media en PRE-E en comparación con mujeres embarazadas sanas, pero con velocidades similares en mujeres no embarazadas..$^{39}$ Los cambios en el tono vascular van a afectar la presión de perfusión cerebral por alteración en la presión de cierre de las arteriolas cerebrales. ${ }^{40}$ Williams y Wilson describieron aumento de la presión de perfusión cerebral en mujeres embarazadas y leve aumento en PRE-E, en comparación con las no embarazadas. ${ }^{41}$

La hipercapnia produce vasodilatación y aumento del flujo sanguíneo cerebral en individuos normales. Shlomit Riskin-Mashiah y col. identificaron que en PRE-E no hay aumento del flujo sanguíneo cerebral al administrar $\mathrm{CO}_{2}$ inhalado. Se piensa que esta respuesta se debe a que los vasos sanguíneos cerebrales se encuentran en vasoconstricción protectora, lo que limita los efectos producidos por el aumento de la presión de perfusión cerebral y daño a la microvasculatura cerebral. ${ }^{4}$ Con estos mecanismos se evita el incremento del flujo sanguíneo cerebral y la sobreperfusión, pero la presión llega a aumentar tanto que lesiona el músculo liso de la pared arterial y se produce vasodilatación segmentaría con lo que aumenta el flujo sanguíneo cerebral y se produce edema cerebral. ${ }^{11,42-44}$ Otros autores consideran que es debido a una alteración de la autorregulación del flujo sanguíneo cerebral, donde 
hay vasoespasmo que produce isquemia y necrosis de las arterias con disrupción de la barrera hematoencefálica lo que genera edema cerebral. ${ }^{45-47}$ Se piensa que la sobreperfusión en algunos casos puede activar focos epileptogénicos en el parénquima cerebral y eso genera una convulsión generalizada. ${ }^{12}$

\section{Predicción de la aparición de PRE-E en el segundo trimestre del embarazo}

Riskin-Mashiah y col. demostraron índices de resistencia y de pulsatilidad bajos en la arteria cerebral media entre las semanas 19 a 28 en quienes después presentaron PRE-E. Valores de índice de resistencia menores a 0.52 en la arteria cerebral media en el segundo trimestre del embarazo tiene una sensibilidad de $80 \%$ y especificidad de $75 \%$ para predecir PRE-E, con valor predictivo positivo y negativo de $62 \%$ y de $88 \% .48$

El estudio de Belfort y col. sugiere que los índices bajos de resistencia en la arteria cerebral media medidos con doppler transcraneal puede predecir la aparición posterior de PRE-E en población étnica homogénea (caucásicos) de bajo riesgo. Se encontraron que valores por debajo de 0.51 de índice de resistencia y de 0.81 de índice de pulsatilidad son útiles para predecir la aparición de la PRE-E, con sensibilidad de $86 \%$ y especificidad de $93 \%$. No está clara la aplicabilidad del doppler transcraneal como tamizaje en el primer trimestre del embarazo, pero en teoría durante este periodo se presenta vasodilatación generalizada con disminución de los índices de resistencia en pacientes que van a presentar PRE-E. Al poder predecir su futura aparición se pueden administrar medicamentos profilácticos como acido acetil salicílico o donadores de óxido nítrico. ${ }^{49}$

\section{Comportamiento del doppler transcraneal en el tercer trimestre con PRE-E}

En un estudio de 40 embarazadas, 20 de las cuales presentaron PRE-E, se les realizó doppler transcraneal el cual demostraba aumento de los índices de resistencia entre las semanas 28 a $32 .{ }^{50}$ Williams y col. identificaron alargamiento del tiempo de duración de la onda sistólica en el doppler transcraneal en preeclampsia del tercer trimestre. ${ }^{51}$ Shlomit Riskin-Mashiah demostró aumento de la presión de perfusión cerebral (90.4 vs $61.9 \mathrm{~mm} \mathrm{Hg}$ ) en PRE-E, con disminución de los índices de resistencia ( 0.46 vs 0.51 ) y de pulsatilidad (0.64 vs 0.76$)$ comparados con embarazadas sanas. ${ }^{11}$

En un estudio realizado por Michael Belfort y col. se identificó aumento de la presión de perfusión cerebral en pacientes con hipertensión arterial y PRE-E sobreagregada en comparación con mujeres con hipertensión arterial, sin encontrar cambios en los índices de resistencia ni de pulsatilidad. Esto apoya la teoría de la sobreperfusión en la fisiopatología de la PRE-E/ eclampsia. ${ }^{12}$

\section{Síndrome HELLP}

El síndrome HELLP se caracteriza por hemólisis, elevación de las enzimas hepáticas y disminución del número de plaquetas. ${ }^{52}$ Williams y Wilson encontraron aumento de las velocidades de flujo de la arteria cerebral media en PRE-E y eclampsia, sin identificar diferencia significativa en la velocidad media de la arteria cerebral media entre pacientes con síndrome HELLP y con PRE-E. ${ }^{53}$ En un estudio se demostró que las pacientes con eclampsia tienen mayor flujo sanguíneo cerebral que aquellas con PRE-E. ${ }^{54}$

\section{Sulfato de magnesio}

Belfort y Moise describieron que cuando se utiliza sulfato de magnesio en PRE-E hay disminución del índice de pulsatilidad. Se cree que este medicamento produce vasodilatación intracraneal de los de pequeño tamaño distantes a la arteria cerebral media. ${ }^{55}$ Estos hallazgos fueron corroborados por Naidu que también encontró disminución de los índices de pulsatilidad cuando se usa sulfato de magnesio en PRE-E. ${ }^{56}$

\section{Conclusiones}

La PRE-E es una enfermedad devastadora que aumenta la morbilidad y mortalidad materna y se asocia 
con retardo del crecimiento intrauterino, por lo que su diagnóstico temprano tiene una importancia radical. Es donde el doppler transcraneal se puede usar como una herramienta que tiene mucha utilidad, sobre todo para predecir su aparición y poder realizar intervenciones terapéuticas a tiempo y un mejor control de las maternas. Faltan estudios del uso del doppler transcraneal en el primer trimestre del embarazo para determinar si tiene algún papel en el diagnóstico temprano de la PRE-E.

\section{Referencias}

1. Wagner LK. Diagnosis and management of preeclampsia. Am Fam Physician. 2004;70(12):2317-24

2. Sibai BM. Diagnosis and management of chronic hypertension in pregnancy. Obstet Gynecol. 1991;78(3 Pt 1):451-61.

3. Sibai BM. Diagnosis and management of gestational hypertension and preeclampsia. Obstet Gynecol. 2003;102(1):181-92.

4. Williams K, Galerneau F. Maternal transcranial Doppler in pre-eclampsia and eclampsia. Ultrasound Obstet Gynecol. 2003;21(5):507-13.

5. Report of the National High Blood Pressure Education Program Working Group on High Blood Pressure in Pregnancy. Am J Obstet Gynecol. 2000;183(1):S1S22.

6. ACOG technical bulletin. Hypertension in pregnancy. Number 219--January 1996 (replaces no. 91, February 1986). Committee on Technical Bulletins of the American College of Obstetricians and Gynecologists. Int J Gynaecol Obstet. 1996;53(2):175-83.

7. Cotton DB, Lee W, Huhta JC, Dorman KF. Hemodynamic profile of severe pregnancy-induced hypertension. Am J Obstet Gynecol. 1988;158(3 Pt 1):523-9.

8. Roberts JM, Taylor RN, Musci TJ, Rodgers GM, Hubel CA, McLaughlin MK. Preeclampsia: an endothelial cell disorder. Am J Obstet Gynecol. 1989;161(5):1200-4.

9. Kublickas M, Lunell NO, Nisell H, Westgren M. Maternal renal artery blood flow velocimetry in normal and hypertensive pregnancies. Acta Obstet Gynecol Scand. 1996;75(8):715-9.

10. Belfort MA, Grunewald C, Saade GR, Varner M, Nisell H. Preeclampsia may cause both overperfusion and underperfusion of the brain: a cerebral perfusion based model. Acta Obstet Gynecol Scand. 1999;78(7):586-91.

11. Riskin-Mashiah S, Saade GR, Herd JA. Cerebrovascular reactivity in normal pregnancy and preeclampsia. Obstet Gynecol. 2001 Nov; 98(5 Pt 1):827-32.

12. Belfort MA, Tooke-Miller C, Allen JC, Varner MA, Grunewald C, Nisell H, et al. Pregnant women with chronic hypertension and superimposed pre-eclampsia have high cerebral perfusion pressure. BJOG. 2001; 108(11):1141-7

13. Horn EH, Filshie M, Kerslake RW, Jaspan T, Worthington BS, Rubin PC. Widespread cerebral ischaemia treated with nimodipine in a patient with eclampsia. $\mathrm{Br}$ Med J. 1990;301(6755):794.

14. Richards A, Graham D, Bullock R. Clinicopathological study of neurological complications due to hypertensive disorders of pregnancy. J Neurol Neurosurg Psychiatry. 1988 Mar; 51(3):416-21

15. Fredriksson K, Lindvall O, Ingemarsson I, Astedt B, Cronqvist S, Holtås S. Repeated cranial computed tomographic and magnetic resonance imaging scans in two cases of eclampsia. Stroke. 1989;20(4):547-53.

16. Osborn AG. Osborn's brain : imaging, pathology, and anatomy. 1st ed. Salt Lake City, Utah: Amirsys; 2004

17. Trommer BL, Homer D, Mikhael MA. Cerebral vasospasm and eclampsia Stroke. 1988; 19(3):326-9.
18. Schatlo B, Pluta RM. Clinical applications of transcranial Doppler sonography. Rev Recent Clin Trials. 2007;2(1):49-57

19. Zwiebel WJ. Zwuebel's Doppler general: introducción a la ultrasonografia vascular. Madrid: Marbán, Elsevier; 2008.

20. Wells PN, Skidmore R. Doppler developments in the last quinquennium. Ultrasound Med Biol. 1985;11(4):613-23.

21. Taylor KJ, Holland S. Doppler US. Part I. Basic principles, instrumentation, and pitfalls. Radiology. 1990;174(2):297-307.

22. Gosling RG. Doppler ultrasound assessment of occlusive arterial disease. Practitioner. 1978;220(1318):599-609.

23. Pourcelot L. Applications cliniques de 1' examen Doppler transcutane. In: Peronneau P, editor. Velocimetrie Ultrasonore Doppler. Paris: INSERM; 1974. p. $780-5$

24. Lindqvist PG, Marsál K, Pirhonen JP. Maternal cerebral Doppler velocimetry before, during, and after a normal pregnancy: a longitudinal study. Acta Obstet Gynecol Scand. 2006; 85(11):1299-303.

25. Easterling TR, Benedetti TJ, Schmucker BC, Millard SP. Maternal hemodynamics in normal and preeclamptic pregnancies: a longitudinal study. Obstet Gynecol. 1990;76(6):1061-9.

26. Bosio PM, McKenna PJ, Conroy R, O>Herlihy C. Maternal central hemodynamics in hypertensive disorders of pregnancy. Obstet Gynecol. 1999;94(6):978-84.

27. Spaanderman ME, Meertens M, van Bussel M, Ekhart TH, Peeters LL. Cardiac output increases independently of basal metabolic rate in early human pregnancy. Am J Physiol Heart Circ Physiol. 2000;278(5):H1585-8

28. Ikeda T, Ikenoue T, Mori N, Nagamachi S, Jinnouchi S, Futami S, et al. Effect of early pregnancy on maternal regional cerebral blood. Am J Obstet Gynecol. 1993 Apr; 168(4):1303-8.

29. Williams K, Wilson S. Maternal middle cerebral artery blood flow velocity variation with gestational age. Obstet Gynecol. 1994;84(3):445-8.

30. Ikeda T, Mori N. Assessment of cerebral hemodynamics in pregnant women by internal carotid artery pulsed Doppler velocimetry. Am J Obstet Gynecol. 1990;163(2):494-8

31. Belfort MA, Tooke-Miller C, Allen JC Jr, Saade GR, Dildy GA, Grunewald C, et al. Changes in flow velocity, resistance indices, and cerebral perfusion pressure in the maternal middle cerebral artery distribution during normal pregnancy. Acta Obstet Gynecol Scand. 2001 Feb; 80(2): p. 104-12

32. Ringelstein EB, Otis SM. Physiological testing of vasomotor reserve. In: Newell DW, Aaslid R, editors. Transcranial Doppler. New York: Raven Press; 1992. p. $83-100$

33. Will AD, Lewis KL, Hinshaw DB Jr, Jordan K, Cousins LM, Hasso AN, et al. Cerebral vasoconstriction in toxemia. Neurology. 1987 Sep; 37(9):1555-7.

34. Lewis LK, Hinshaw DB Jr, Will AD, Hasso AN, Thompson JR. CT and angiographic correlation of severe neurological disease in toxemia of pregnancy. Neuroradiology. 1988; 30(1):59-64

35. Zatik J, Aranyosi J, Settakis G, Páll D, Tóth Z, Limburg M, et al. Breath holding test in preeclampsia: lack of evidence for altered cerebral vascular reactivity. Int J Obstet Anesth. 2002;11(3):160-3.

36. Demarin V, Rundek T, Hodek B. Maternal cerebral circulation in normal and abnormal pregnancies. In: T R, editor. Scand Acta Obstet Gynecol Scand. 1997 Aug;76(7):619-24.

37. Steegers EA, von Dadelszen P, Duvekot JJ, Pijnenborg R. Pre-eclampsia. Lancet. 2010;376(9741):631-44

38. Zeeman GG. Neurologic complications of pre-eclampsia. Semin Perinatol. 2009;33(3):166-72.

39. Sherman RW, Bowie RA, Henfrey MM, Mahajan RP, Bogod D. Cerebral haemodynamics in pregnancy and pre-eclampsia as assessed by transcranial Doppler ultrasonography. Br J Anaesth. 2002;89(5):687-92.

40. Belfort MA, Saade GR, Yared M, Grunewald C, Herd JA, Varner MA, et al. Change in estimated cerebral perfusion pressure after treatment with nimodip- 
ine or magnesium sulfate in patients with preeclampsia. Am J Obstet Gynecol. 1999;181(2):402-7.

41. Williams KP, Wilson S. Variation in cerebral perfusion pressure with different hypertensive states in pregnancy. Am J Obstet Gynecol. 1998 Nov; 179(5):1200-3

42. Hauser RA, Lacey DM, Knight MR. Hypertensive encephalopathy. Magnetic resonance imaging demonstration of reversible cortical and white matter lesions. Arch Neurol. 1988 Oct; 45(10):1078-83

43. Chester EM, Agamanolis DP, Banker BQ, Victor M. Hypertensive encephalopathy: a clinicopathologic study of 20 cases. Neurology. 1978; 28(9 Pt 1):928-39.

44. Strandgaard S, Paulson OB. Cerebral autoregulation. Stroke. 1984;15(3):413-6.

45. Belfort MA, Saade GR, Grunewald C, Dildy GA, Varner MA, Nisell H. Effects of blood pressure on orbital and middle cerebral artery resistances in healthy pregnant women and women with preeclampsia. Am J Obstet Gynecol. 1999;180(3 Pt 1):601-7.

46. Redman CW. Eclampsia still kills. Br Med J (Clin Res Ed). 1988 Apr 30; 296(6631):1209-10.

47. Aaslid R, Markwalder TM, Nornes H. Noninvasive transcranial Doppler ultrasound recording of flow velocity in basal cerebral arteries. J Neurosurg. 1982;57(6):769-74

48. Riskin-Mashiah S, Belfort MA, Saade GR, Herd JA. Transcranial doppler measurement of cerebral velocity indices as a predictor of preeclampsia. Am J Obstet Gynecol. 2002;187(6):1667-72
49. Belfort M, Van Veen T, White GL, Kofford S, Allred J, Postma I, et al. Low maternal middle cerebral artery Doppler resistance indices can predict future development of pre-eclampsia. Ultrasound Obstet Gynecol. 2012;40(4):406-11.

50. Zunker P, Happe S, Georgiadis AL, Louwen F, Georgiadis D, Ringelstein EB. Maternal cerebral hemodynamics in pregnancy-related hypertension. A prospective transcranial Doppler study. Ultrasound Obstet Gynecol. 2000 Aug; 16(2):179-87.

51. Williams KP, Moutquin JM. Do maternal cerebral vascular changes assessed by transcranial Doppler antedate pre-eclampsia?. Ultrasound Obstet Gynecol. 2004;23(3):254-6.

52. Martin JN, Blake PG, Perry KG, McCaul JF, Hess LW, Martin RW. The natural history of HELLP syndrome: patterns of disease progression and regression. Am J Obstet Gynecol. 1991;164(6 Pt 1):1500-9; discussion 9-13

53. Williams KP, Wilson S. Maternal middle cerebral artery velocity changes in HELLP syndrome versus pre-eclampsia. Ultrasound Obstet Gynecol. 1998 Mar; 11(3): 195-8

54. Williams KP, Wilson S. Maternal cerebral blood flow changes associated with eclampsia. Am J Perinatol. 1995;12(3):189-91.

55. Belfort MA, Moise KJ Jr. Effect of magnesium sulfate on maternal brain flow in pre-eclampsia: a randomized, placebo-controlled study. Am J Obstet Gynecol. 1992 Sep; 167(3):661-6.

56. Naidu S, Payne AJ, Moodley J, Hoffmann M, Gouws E. Randomized study assessing the effect of phenytoin and magnesium sulphate on maternal cerebral circulation in eclampsia using transcranial Doppler ultrasound. Br J Obstet Gynaecol. 1996 Feb; 103(2):111-6. 